\title{
PAROXYSMAL TRIGEMINAL PAIN WITH TUMOURS OF THE NERVUS AGUSTIGUS *
}

\author{
By
}

\author{
HARRY L. PARKER, Dublin
}

IN 1910 Weisenburg ${ }^{1}$ published a communication under the title ' Cerebellopontine tumour diagnosed for six years as tic douloureux.' This was the first recorded case of a particular type of pain associated with acoustic neurofibroma. The next important phase in the study of this particular tumour was Cushing's ${ }^{2}$ book Tumours of the Nerous Acusticus in 1917. In the series Cushing reported including $\mathbf{3 0}$ cases of the disease there were only four cases where there was any complaint of pain at all. In none of them was pain conspicuous, persistent, or in any way like that associated with tic douloureux. In $1928 \mathrm{I}^{3}$ made a study of 53 cases of tumours of the acoustic nerve. Only those cases were taken in which the diagnosis had been established by microscopic study of tumour tissue obtained either at operation or at necropsy. In each case there had been a neurological examination and a careful study to determine the degree of involvement of the fifth nerve. In only four cases in the entire series was there any disturbance of sensation which could be described as pain. In only one of these four was the pain a dominant and persistent symptom and one which played the major part in the patient's complaint of symptoms. In the remaining three pain was only transitory and not conspicuous during the course of illness.

In the case of the one patient who had persistent pain the interesting feature was that the type of pain corresponded exactly to the syndrome known as tic douloureux. The earlier signs of deafness and nystagmus were inconspicuous, and of more interest still was the fact that the pain became relieved by the usual treatment for tic douloureux, namely, injection of the nerve with alcohol. It was only later with the advent of vomiting, ataxia and vertigo, that the true nature of the patient's condition was appreciated. To this experience, two more examples may be added.

A man, age 46, was seen by me in April, 1936, because of pain in the left upper and lower jaws. He stated that the previous seven years he had had recurrent attacks of fainting and vomiting. On one occasion he had been unconscious for from one to two hours, but there were no convulsive movements during the unconscious period. Three years before he had noticed a ringing and buzzing sound in the left ear associated with a progressive deafness. A few months later he complained of

* Read before the Association of British Neurologists, June 12-13, 1936. 
darting and stabbing pains in the canine tooth of the left upper jaw. Removal of the tooth produced no relief, and touching the lip or gum in the same region would initiate a paroxysm of pain. More teeth were removed without apparent benefit. He had remissions and exacerbations of this difficulty up to the time of his examination, and on one occasion was free from pain for at least eight months. A year before examination the pain spread from the upper jaw into the lower, and talking, eating, shaving, or washing his face when the pain was present would initiate a paroxysm. The paroxysms never lasted longer than 60 seconds, and pressure on the lower jaw seemed to produce relief.

During the six months previous to his examination many other symptoms appeared. He had attacks of vertigo, paroxysmal in character, and during at least a few of these attacks he fell to the ground. During the attacks of vertigo he had double vision, but a few weeks prior to his examination his vision had become generally poor, and he could not recognize people across the street. His gait was becoming progresively unsteady so that he walked as if he was drunk, and headaches appeared chiefly in the occipital region. With all these complaints his chief concern was the pain in his upper and lower jaws.

He was a well-built, burly, florid-complexioned man who presented himself with a reeling, staggering gait. There was complete deafness in the left ear and the corneal reflex was absent on the left side. There was nystagmus on lateral gaze more marked to the left than to the right, and both optic nerve heads were swollen-about one and one-half diopters on the right side and one diopter on the left. There was a slight hypæsthesia on the left side in the second and third divisions of the left nerve. The finger-nose test showed incoordination of both upper extremities, more marked on the left side. The same was true of both lower extremities, and his gait was ataxic with a certain degree of tilting of his head to the left side. The plantar reflex on the left side was extensor in character.

The diagnosis was made of left acoustic neurofibroma, and operation was advised. The patient did not consent to this treatment and left for home.

Pain in the area supplied by the fifth nerve was the dominant complaint of this patient. Similar to the previous case described by me, other complaints of vertigo, ataxia, deafness, and tinnitus seemed of less importance. Generally speaking, the case was otherwise a typical one of acoustic neurofibroma. Alcohol injection was not advised, for it scemed more important to attack the primary difficulty.

A woman, age 52, was examined in IIarch, 1936. Her chief difficulty was attacks of pain in the right side of her face. As a secondary complaint, she had marked difficulty in walking, due to ataxia. It was difficult to obtain a coherent history because of repeated attacks of severe pain, making talking laborious. About tive years before she had noticed deafness in her left ear, and with this there were headaches and staggering. At the hospital where she was examined she was given a course of X-ray treatments to her head. This relieved the headaches, but she complained that her gait was becoming more and more unsteady, and this continued during the years that followed. Six months previously, however, the pain in her face began, and from then on this was her chief complaint. The pain was located just above and to the lateral side of the right angle of her mouth. From there it radiated upwards into the right eye and right temporal region. The pain was sudden, sharp, severe, and of a burning character. It was initiated by movement of any kind, and while the paroxysms of pain were short in duration, she dreaded their occurrence so much that she lay for days immobile in bed. She preferred to keep her eyes closed, for she 
found that raising the upper lid started the paroxysms. She had occasional remissions of pain of no longer than a week in duration.

At the time of the examination she was confined to the bed, and she was having one paroxysm of pain after another. She seemed to think that opening her eyes increased the pain, but with a little patience there could be established a nystagmus in all directions. Corneal sensation could not be tested because of the blepharospasm and pain. She was completely deaf in the left ear, but there was no facial weakness. The right patellar reflex was greater than the left, but plantar responses were flexor in character. There was no anesthesia of the face, and the fundi were normal. After some persuasion she consented to walk, and there was a marked ataxia with a tendency to hold the head tilted to the left. The finger-nose test showed incoordination in both hands, more marked on the left side. The same was true in similar tests applied to both lower extremities.

The diagnosis was difficult because of the poor cooperation of the patient. Disseminated sclerosis and accustic neurofibrcma were considered. In the hope of securing better cooperation, relief of pain was obtained by injecting the second and third divisions of the right fifth nerve with alcohol. This was successful and allowed a more satisfactory cxamination of the patient. As an additional finding it was discovered that the left corneal reflex was abolished. The injection performed on the right side did not ruin the corneal reflex on that side. It was then considered that a diagnosis of left-sided acoustic neurofibrcma was correct and surgical exploration oi the left cerebellopontine angle was advised. On April 16, 1936, Mr. McConnell operated and found a large acoustic neurofibroma on the left side, and as far as he was able to see the right cerebellopontine angle was intact. The tumour was incised and the core removed by suction. Unfortunately, death from complications occurred 18 days later. Necropsy was performed and the remains of a left-sided acoustic ncurofibroma were found. The tumour was quite large and had decply indented the left side of the pons. More important still was the marked distortion of the brainstem so that the pons and medulla were pushed far to the right of the midline and evidently compression of the right side of the pons against the petrous portion of the temporal bone had taken place.

\section{DISCUSSION}

These two clinical cases are reported for two main reasons : firstly, to add to the clinical syndrome of acoustic neurofibroma, and, secondly, in the hope that some light may be thrown on the actual mechanism involved in the production of a certain type of trigeminal pain.

In this first category, argument might arise that the cccurrence of this type of pain identical with that of tic douloureux represents a mere coincidence. A moment's reflection would show that this is not so. Both tic douloureux and acoustic neurofibroma are rare diseases and their simultaneous appearance in the course of a pathological process involving the fifth nerve apparatus is beyond the bounds of coincidence. The appearance of pain on the opposite 
side to the tumour ceases to be so extraordinary when the necropsy material is considered and it is realized that the pons was not merely compressed on the side of the tumour but damaged in its entirety by the change in its whole normal anatomical relationships. Cases enough have been published of areflexia cornealis and anæsthesia in the domain of the fifth nerve on the opposite side to the tumour to make the more rare appearance of paroxysmal pain, also on the opposite side, not so devoid of explanation. So far as my knowledge is concerned, while cases have been recorded of bilateral objective fifth nerve damage no mention has been made of bilateral paroxysmal pain during the course of acoustic neurofibroma.

The second and more interesting point in these cases is that the pain was identical in all respects with that seen in tic douloureux, a disease wherein the pathological cause is, up to date, entirely unknown. Another disease of very different character, namely, disseminated sclerosis, will at times produce the same pain syndrome and frequently it is bilateral. Two cases 4,5 have been recorded of this disease with necropsy wherein a sclerotic plaque was found at the root entrance zone of the fifth nerve on the side of the pain. Further it has been observed that in all three diseases-tic douloureux, acoustic neurofibroma, and disseminated sclerosis-the pain is relieved by injection of the peripheral branches of the fifth nerve with alcohol. In one case of disseminated sclerosis reported by me ${ }^{5}$ relief was obtained by surgical section of the posterior root. The inference from this is obvious, and that is that the explosion of pain depends entirely on incoming trigger-like stimuli and pain ceases when there is a dead and non-functioning afferent pathway.

The pathological changes found up to date in cases of tic douloureux are either nil or not sufficiently explanatory as to the essential cause of the disease. Harris ${ }^{6}$ has suggested very reasonably that septic neuritis of the terminal filaments of the fifth nerve in contact with diseased teeth and sinuses is a prominent factor in the production of the disease. He noted, very early, that the second and third divisions and just the branches more in contact with sepsis are the first to be affected and that first divisional involvement is late or not at all. In cases where the teeth are sound, Harris assumes a previous infection and caries in the milk teeth. Again, this particular pain syndrome is practically confined to the fifth and ninth cranial nerves, the latter in contact continuously with tonsillar sepsis. Harris also invokes heredity and trauma as possible factors.

There are points militating against an unreserved acceptance of the above as the whole explanation of the pain mechanism. Dental, tonsillar, and sinus infections in this day and age are excessively common, and while tic douloureux is rare, glossopharyngeal neuralgia is even more so, in spite of the fact that tonsillar sepsis is almost universal at certain ages. Hereditary influences cannot be invoked since direct transmission of tic douloureux from parent to child is a very rare observation, and to fall back on some special constitutional predisposition is begging the question. Again, it is well known 
that nerves may be bathed in pus, as in osteomyelitis of the jawbone or elsewhere, and no neuritis appears, and it has been observed frequently that peripheral nerves are highly resistant to contiguous septic processes. Dental caries in Great Britain is extremely common and much less so in the United States, and yet to my limited observation the incidence of tic douloureux seems about the same. Finally, to assume a septic neuritis as the sole cause of the particular type of pain syndrome under discussion one would wish for more evidence such as numbness, reflex and trophic changes in at least the majority of cases of tic douloureux, as well as the pain which is usually the solitary symptom of the disease.

In two diseases such as acoustic neurofibroma and disseminated sclerosis the gross pathology is known. In tic douloureux there is no manifest pathology. It might be reasonable to assume that all three diseases of very different actiology nust work through a common factor to produce so identical a pain syndrome. Already it has been mentioned that cutting the posterior root of the fifth nerve in disseminated sclerosis stops the pain. Of more interest is the case mentioned by Cairns ${ }^{7}$ wherein a case of acoustic neurofibroma with paroxysmal trigeminal pain was operated on and the tumour removed. The pain did not cease, however, and second operation to cut the posterior root was necessary and was then successful. Foerster, ${ }^{8}$ to explain such a pain syndrome, suggests a state of functional hyperexcitability in the nerve apparatus caused by many and different influences. All incoming stimuli in such cases are responded to by an explosion of pain. This hyperexcitability may wax and wane with the fluctuations of the disease; its remissions and exacerbations and finally the patient's miseries can be put an end to by blocking all incoming stimuli with alcohol or by surgery. Septic infections along the nerve pathway, trauma, vascular disease, sclerosis of the root entrance zone, tumours contiguous to the nerve root and pressure on the side of the pons may each be the causal factor in any given case, and all work through a common mechanism, namely, creating in some way a state of exaggerated irritability of the ninth and fifth nerve apparatus and for some unknown biological reason largely confined to these two cranial nerves. Once this state is induced it is possible that a conditioned reflex is laid down and facilitation occurs, making it more easy each time for pain to be produced. As an analogy, but occurring in the motor system, one thinks of facial spasm, a functional condition of increased irritability of the seventh cranial nerve. This has an insidious beginning, but once the motor activity appears it spreads to include all the areas supplied by the seventh nerve and is permanent. It has, however, the same fluctuation in degree as tic douloureux and is likewise relieved by blocking the nerve with alcohol. Again, nothing of quite the same nature is seen in the nerves of the trunk or extremities, and as a further analogy in one of Cushing's earlier cases of acoustic neurofibroma a facial spasm appeared, and in one case of my own a similar phenomenon occurred during the growth of the tumour. Facial spasm often makes its 
appearance after recovery from a mild Bell's palsy. As the facial muscles recover their power, instead of normal activity, there is overactivity and spasm. A similar situation with the fifth nerve is recorded by Harris. ${ }^{9}$ In a group of patients of his and for no obvious reason anæsthesia of the fifth nerve area appeared. Following the spontaneous disappearance of anæsthesia, a tic douloureux syndrome appeared and persisted. While incoming afferent stimuli start a pain in tic douloureux, corresponding efferent impulses exaggerate and intensify facial spasm. Facial spasm may be bilateral, and this is just as rare as bilateral tic douloureux, but a case published by me ${ }^{10}$ as paraspasmus facialis was really a bilateral facial spasm. The age-incidence of facial spasm is similar to that of tic douloureux, and in all probability the underlying mechanism of the two diseases is identical and rests in the state of hyperexcitability of a cranial nerve, conditioned and facilitated by one cause or another. At first partial, transient, and separated by intervals of remission, it later involves all the branches of the nerve, and becoming constant in character, it is more and more readily induced by afferent or efferent stimuli.

\section{REFERENCES}

1 Weisenburg, 'T. H., 'Cerebello-pontine tumour diagnosed for six years as tic douloureux,' Jour. Amer. Med. Assoc., 1910, 84, 1600.

2 Cusnisg, H., Tumours of the Nervus Acusticus, W. B. Saunders, Philadelphia, $1917,296$.

3 Parker, H. L., 'Tumours of the nervus acusticus. Signs of involvement of the fifth cranial nerve,'Arch. Neurol. and Psychiat., 1928, 20, 309.

4 Oppexhem, H., Text Book of Nercous Diseases, 5th ed., New York, G. E. Stechart \& Co., 1911, 33\%.

5 Parker, H. L., 'Trigeminal neuralgic pain associated with multiple sclerosis,' Brain, 1928, 51, 46.

- Harris, W., Neuritis and Neuralgia, Humphrey Milford, Oxford University Press, 1926.

7 Cairns, H. Personal communication.

8 Foerster, O., Die Leitungsbahnen des Schmerzgefühls, Berlin, 1927.

9 Harris, W., 'Paroxysmal neuralgic tic as a sequel of trigeminal neuritis,'Brit. Med. Jour., 1935, 1, 1112.

10 Parker, H. L., 'Bilateral facial spasm,'Amer. Jour. Ophthalmol., 1932, 16. 